\title{
Simultaneous Determination of Large Pretilt Angles and Cell Gap in Liquid Crystal Displays
}

\author{
Y. W. Li, Jacob Y. L. Ho, Fion S. Y. Yeung, and H. S. Kwok, Fellow, IEEE
}

\begin{abstract}
A method for the measurement of arbitrary pretilt angles in liquid crystal displays is proposed and demonstrated. The cell gap can also be determined accurately in the same experiment as well. This method makes use of a photo-elastic modulator and is capable of measuring pretilt angles from $0^{\circ}$ to $90^{\circ}$ precisely.
\end{abstract}

Index Terms-Cell gap, measurement, pretilt angle.

\section{INTRODUCTION}

$\mathbf{P}$ RETILT angle is an important parameter in the design of liquid crystal displays (LCDs). Traditionally, pretilt angles are limited to a few degrees from the normal $\left(90^{\circ}\right)$ or horizontal $\left(0^{\circ}\right)$ directions. However, with the advent of new alignment techniques, large pretilt angles of $10^{\circ}-80^{\circ}$ are now routinely available [1]. Many important applications are possible based on these large pretilt angles. For example, large pretilt angles can be utilized to decrease the response time of LCD for video and field-sequential color applications [2], [3]. Large pretilt angles are also important in the production of o-plates and other wide-view angle compensation films [4]. Also, large pretilt angles are needed for a class of bistable nematic-mode LCDs [5], [6].

All of the applications mentioned require a high pretilt angle from $10^{\circ}$ to $80^{\circ}$. Therefore, it is important to control and measure the pretilt angle accurately. It is well known that the conventional crystal rotation method [7] is not accurate for large pretilt angles, because the shift of the center of the fringe pattern is too large. For pretilt angles higher than $15^{\circ}$, the magnetic null method is commonly used. However, it is quite complicated and a huge magnet is needed. As well. the time required for such a measurement is long. Therefore, a simpler and more rapid technique is required. Several other techniques have been proposed [8], [9]. However, they all have difficulty measuring LCDs with a small cell gap ranging from 1 to $5 \mu \mathrm{m}$.

\section{THEORY AND METHOD OF MEASUREMENT}

We propose a simple method to measure large pretilt angles accurately for small cell gap LC cells. In addition, this method can also yield an accurate value of the cell gap ranging from 1 to $5 \mu \mathrm{m}$. Instead of a full off-axis transmission scan $\left(-60^{\circ}\right.$ to $\left.60^{\circ}\right)$, this method only requires one on-axis $0^{\circ}$ and one off-axis i.e.,

Manuscript received January 9, 2007; revised April 5, 2007. This work was supported by the Hong Kong Government Innovations and Technology Fund.

The authors are with the Center for Display Research, Department of Electronic and Computer Engineering, Hong Kong University of Science and Technology, Hong Kong (e-mail: eekwok@ust.hk).

Digital Object Identifier 10.1109/JDT.2007.901565

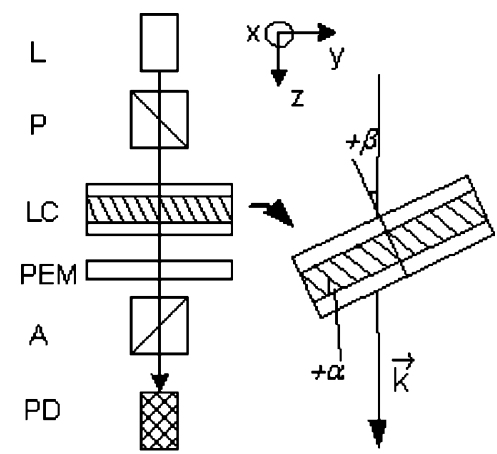

(a)

(b)

Fig. 1. Optical setup of the proposed method. (a) Overview. (b) Geometry of the LC cell.

$60^{\circ}$, incident angle to obtain the corresponding pretilt angle and cell gap accurately. The optical setup can easily be extended or modified to determine the pretilt angle of reflective-mode LCD or the spatial variation of the pretilt angle and cell gap by using a high-resolution CCD camera.

Fig. 1(a) shows the optical setup for the measurement. $\mathrm{L}$ is a 632.8-nm helium neon laser. PEM represents the photo-elastic modulators. $\mathrm{LC}$ is the test cell which is placed on a rotational stage. $\mathrm{P}$ and $\mathrm{A}$ are the polarizer and analyzer, respectively. They are $+45^{\circ}$ and $-45^{\circ}$ with respect to the liquid crystal alignment direction. PD is the photo detector. A beam of polarized light enters the LC cell at an oblique angle, as shown in Fig. 1(b). The test cell is rotated about the $x$-axis and the laser ray incident angle is named as $\beta$. The pretilt angle of the liquid crystal is $\alpha$

The effective retardation of an LCD at an off-axis situation has been analyzed in the past. The generalized $2 \times 2$ matrix formulations for birefringent media have been obtained at oblique incidence [10], [11]. Yu et al. [12] gave a comparison of the different approaches. Here, we follow the approach of Lien [11]. We consider the LC test cell is homogenously aligned with a uniform tilt angle. The retardation is given by

$$
\begin{aligned}
\Gamma(\alpha, \beta, d)= & \frac{2 \pi d}{\lambda}\left(\sigma_{e z}-\sigma_{o z}\right) \\
\sigma_{\mathrm{ez}}= & \frac{\left(n_{e}^{2}-n_{o}^{2}\right) \sin 2 \alpha \sin \beta}{2\left[n_{o}^{2}+\left(n_{e}^{2}-n_{o}^{2}\right) \sin ^{2} \alpha\right]} \\
& +\frac{n_{o} n_{e} \sqrt{n_{o}^{2}+\left(n_{e}^{2}-n_{o}^{2}\right) \sin ^{2} \alpha-\sin ^{2} \beta}}{n_{o}^{2}+\left(n_{e}^{2}-n_{o}^{2}\right) \sin ^{2} \alpha} \\
\sigma_{o z}= & \sqrt{n_{o}^{2}-\sin ^{2} \beta}
\end{aligned}
$$




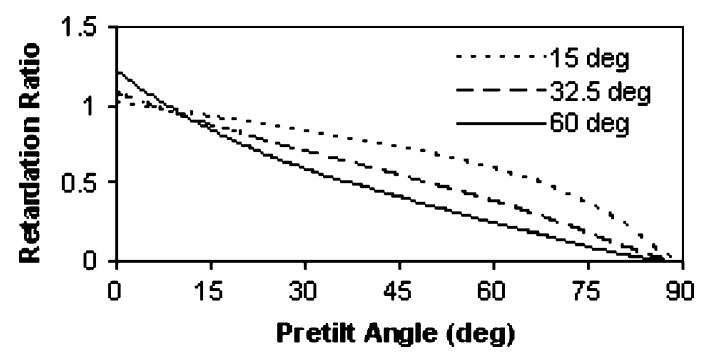

Fig. 2. Retardation ratio at different pretilt angles.

where $\Gamma$ is the retardation of the LC cell, $d$ is the cell gap, $\lambda$ is the wavelength of the incident light, $\alpha$ is the pretilt angle, and $\beta$ is the laser incident angle. Also $n_{e}$ and $n_{o}$ are the extraordinary and ordinary refractive indices of LC, respectively. $\sigma_{e z}$ and $\sigma_{o z}$ are two eigenvalues in the uniaxial medium.

In principle, from (1), if the retardation is measured for two angles of incidence $\beta_{1}$ and $\beta_{2}$, both $\alpha$ and $d$ can be determined. By eliminating the cell gap variable, one obtains the ratio of retardation $\chi$ as

$$
\chi\left(\alpha, \beta_{1}, \beta_{2}\right)=\frac{\Gamma\left(\alpha, \beta_{1}\right)}{\Gamma\left(\alpha, \beta_{2}\right)}=\frac{\sigma_{\mathrm{ez} 1}-\sigma_{\mathrm{oz} 1}}{\sigma_{\mathrm{ez} 2}-\sigma_{\mathrm{oz} 2}} .
$$

From (4), the value of $\alpha$ can be calculated readily. After obtaining the pretilt angle, the corresponding cell gap $d$ can be calculated as

$$
d=\frac{\Gamma_{M} \lambda}{2 \pi\left(\sigma_{e z}-\sigma_{o z}\right)} .
$$

Here, $\Gamma_{M}$ is the measured retardation.

Fig. 2 shows all possible retardation ratios corresponding to pretilt angles from $0^{\circ}$ to $90^{\circ}$, with $n_{e}=1.71$ and $n_{o}=1.5076$. $\beta_{1}$ is assumed to be $0^{\circ}$ and the three different values of $\beta_{2}$ are indicated in the Fig. 2. The change of the retardation ratio is almost proportional to the pretilt angles when $\beta_{2}=32.5^{\circ}$ (long dashed line). Every pretilt angle (from $0^{\circ}$ to $90^{\circ}$ ) is uniquely defined by one retardation value as well.

\section{SEnsitivity AND Tolerance OF MEASUREMENT}

The sensitivity $S$ of the proposed method can be estimated by $S=|d \chi / d \alpha|$ According to the geometry in Fig. 1(b), one can always obtain higher retardation value at $+\beta$ than that at $-\beta$. It is because the effective extraordinary eigenvalue $\sigma_{e z}$ in (2) is larger at $+\beta$. If the situation is reversed, the position of the test cell must be reversely placed and the pretilt angle becomes negative $-\alpha$, By using such a simple procedure, it can be ensured that the test cell is placed properly according to Fig. 1(b) and the pretilt angle is always found positive. Fig. 3(a) shows the minimum sensitivity is $0.63 \mathrm{rad}^{-1}$ at $52^{\circ}$. The maximum sensitivity is $0.85 \mathrm{rad}^{-1}$ at $0^{\circ}$ and $80^{\circ}$. Obviously, different incident angles set $\left(\beta_{1}, \beta_{2}\right)$ will result in different sensitivity behavior with respect to the pretilt angles. This can be optimized by means of selecting the proper incident angle set. However, the value of the sensitivity is quite small and the accuracy of this method is quite good already.
The tolerance of measurement is also studied. It is assumed that the accuracy of the measurement must be smaller than $\pm 1^{\circ}$. Based on this assumption, the corresponding maximum deviation, $\varepsilon$, allowed during the measurement is estimated by

$$
\chi_{o}\left(\frac{1+\varepsilon}{1-\varepsilon}\right)-\chi_{o}=\Delta \chi \rightarrow \varepsilon=\frac{\Delta \chi}{2 \chi_{o}+\Delta \chi}
$$

where $\Delta \chi=S \cdot \Delta \alpha, \Delta \alpha=1^{\circ}$ and $\chi_{o}=\Gamma\left(\alpha, \beta_{1}\right) / \Gamma\left(\alpha, \beta_{2}\right)$.

Fig. 3(b) shows the tolerance for different pretilt angles. When $\beta_{1}=0^{\circ}$, larger $\beta_{2}$ gives larger tolerance (maximum $>50 \%$ ). It also proves that sensitivity is not the most crucial factor in our measurement. At $\beta_{2}=60^{\circ}$, the worst case of the tolerance is $1.13 \%$ at a pretilt angle of $21^{\circ}$.

To measure retardation values, several methods are available, such as the Soleil-Babinet compensator, phase detection of Young's fringes, and photoelastic modulator (PEM) [13]-[16]. PEMs have several unique features such as wide spectral range, large aperture, wide acceptance angle, and high precision of phase modulation. The PEM is typically used for fast high-sensitivity measurements. According to Oakberg et al. [14], the general expression for the intensity function received by the photodiode is given by

$$
\begin{gathered}
I_{D}=\frac{1}{2}\left[1-\cos (\Gamma) J_{0}\left(A_{0}\right)+2 \sin (\Gamma) J_{1}\left(A_{0}\right) \cos (\omega t)\right. \\
\left.+2 \cos (\Gamma) J_{2}\left(A_{0}\right) \cos (2 \omega t)+\cdots\right]
\end{gathered}
$$

where $A_{0}=2.4048$ is the PEM retardation amplitude, $\omega=50 \mathrm{kHz}$ is the angular frequency of the modulator, and $J_{0}\left(A_{0}\right), J_{1}\left(A_{0}\right)$, and $J_{2}\left(A_{0}\right)$ are the Bessel functions. The latter expression shows the dc component of the expression and the first and second harmonics of the optical intensity signals. Using the reference signals from the modulator controller, the first $V_{1 f}$ and second $V_{2 f}$ harmonics can be detected with a lock-in amplifier [8], [9]. Thus

$$
\begin{aligned}
& V_{1 f}=2 K \sin \left(\Gamma J_{1}\left(A_{o}\right)\right) \\
& V_{2 f}=2 K \cos \left(\Gamma J_{2}\left(A_{o}\right)\right)
\end{aligned}
$$

where $K$ is an instrument constant and is of no consequence. The retardation of the LC cell can be obtained as follows. For $0 \leq \Gamma \leq \pi \Rightarrow V_{1 f} \geq 0$

$$
\Gamma=\frac{\pi}{2}-\tan ^{-1}\left[\frac{V_{2 f}}{V_{1 f}} \frac{J_{1}\left(A_{0}\right)}{J_{2}\left(A_{0}\right)}\right] .
$$

For $\pi \leq \Gamma \leq 2 \pi \Rightarrow V_{1 f} \leq 0$

$$
\Gamma=\frac{3 \pi}{2}-\tan ^{-1}\left[\frac{V_{2 f}}{\left|V_{1 f}\right|} \frac{J_{1}\left(A_{0}\right)}{J_{2}\left(A_{0}\right)}\right] .
$$

In order to test whether the measurement system fulfills the requirement of designed tolerance $\sim 1 \%$, the noise level of the system is measured. We did an experiment with an empty cell. Fig. 4 shows the retardations fluctuation over 100 measurements. Sampling rate is 0.2/s. From this plot, the root mean square measurement fluctuation is less than $<0.01 \mathrm{~nm}$. The 


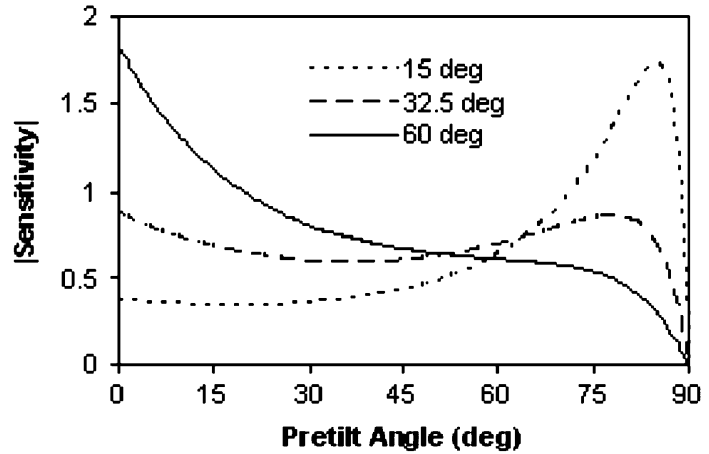

(a)

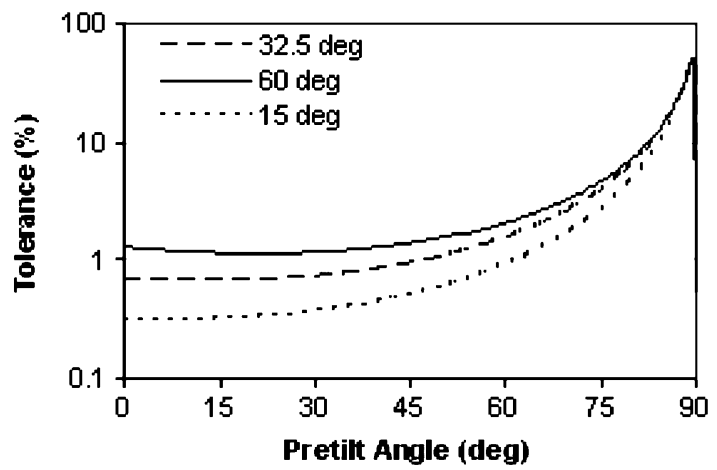

(b)

Fig. 3. Accuracy of the measurement method. (a) Measurement sensitivity. (b) Tolerance (\%).

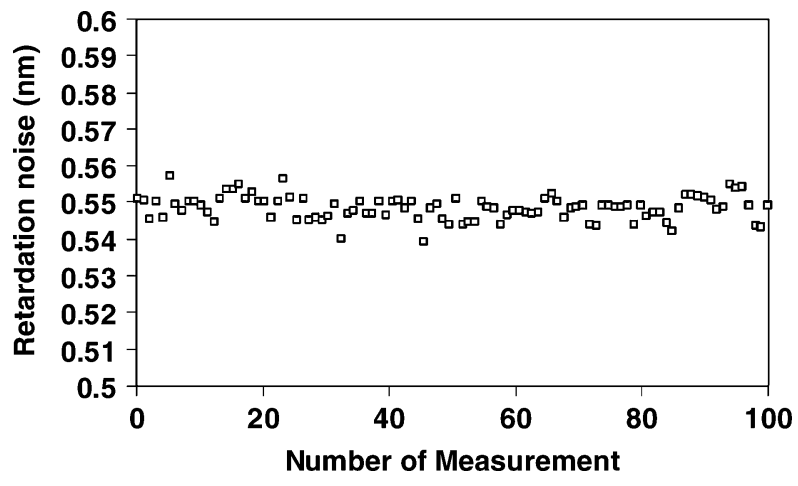

Fig. 4. Noise data for the measurement system.

system noise is mainly due to optical alignment error, temperature fluctuation, and residual birefringence.

We assume that the system induces a $0.1-\mathrm{nm}$ error, which is ten times the system fluctuation. Since the smallest retardation of the LC cell is about $100 \mathrm{~nm}$, the measurement error is less than $0.1 \%$. This is acceptable for most industrial and scientific applications.

\section{EXPERIMENTS AND RESULTS}

In order to verify the proposed measurement method, several homogenous cells (A, B, C, and D) with different pretilt angles were fabricated. The liquid crystal is MLC-6080 from Merck. The optical parameters are as follows: $\mathrm{n}_{e}=1.71, \mathrm{n}_{o}=1.5076, \mathrm{~K}_{11}=14.4 \mathrm{pN}, \mathrm{K}_{22}=7.1 \mathrm{pN}$, and $\mathrm{K}_{33}=19.1 \mathrm{pN}$. Their retardations at different incident angles are shown in Fig. 5.

The pretilt angles and cell gap for the test cells were obtained by using the incident angle set $\left(0^{\circ}, 60^{\circ}\right)$ (see Table I).

The transmission intensity against different off-axis incident angles of the test cells is measured in Fig. 6(a)-(d). The intensity of the test cells are periodic function. By making use of this effect, the accuracy of the measured result can be verified. Based on the measured pretilt angles and cell gaps from Table I, the position of those local maxima and local minima are first estimated. Such results are compared with the values found experimentally. According to Cuminal et al. [7], when the polarizer

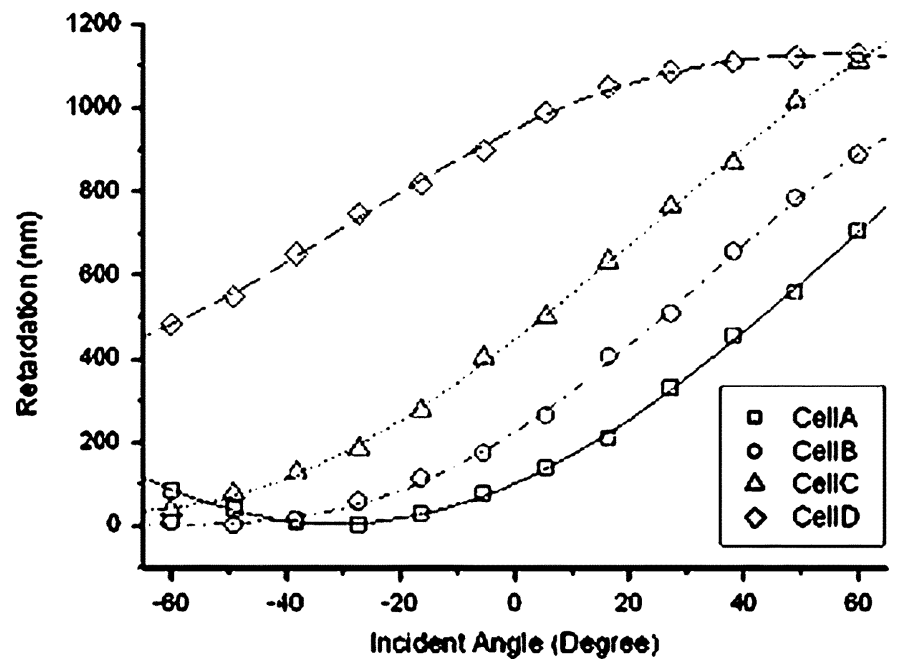

Fig. 5. Measured retardation value for samples A, B, C, and D.

TABLE I

MeAsured PARAmeters For the Four Cells $\left(\beta_{1}=0^{\circ}, \beta_{2}=60^{\circ}\right)$

\begin{tabular}{lll}
\hline $\mathrm{A}$ & 70 & 5.08 \\
\hline $\mathrm{B}$ & 58 & 5.33 \\
\hline $\mathrm{C}$ & 44 & 5.08 \\
\hline $\mathrm{D}$ & 15 & 5.39 \\
\hline
\end{tabular}

and the analyzer are $+45^{\circ}$ and $-45^{\circ}$ with respect to the director of liquid crystal, the intensity $I$ of the cells is given by

$$
I=\frac{1}{2} \sin ^{2}\left[\frac{\pi}{\lambda}\left(\sigma_{e z}-\sigma_{o z}\right) d\right] .
$$

Cuminal et al. also stated that the reflection between interfaces of air/glass/ITO/PI/LC depends on the incident angle. Therefore, the absolute intensity will become lower, especially at large off-axis incident angles. However, since the position of the maxima and minima of the curve will not be altered by reflections, we can ignore such effects in the determination of maxima and minima.

Fig. 6(a) and (b) shows the experimental results of cells A, $\mathrm{B}, \mathrm{C}$, and D, respectively. The local minima and maxima of the 


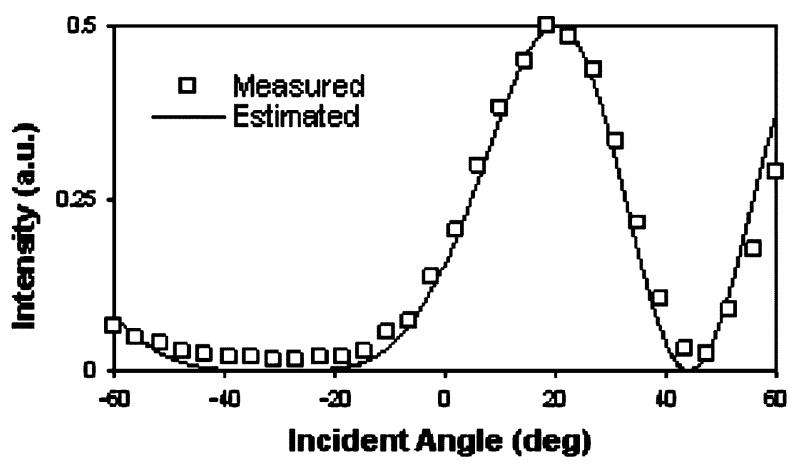

(a)

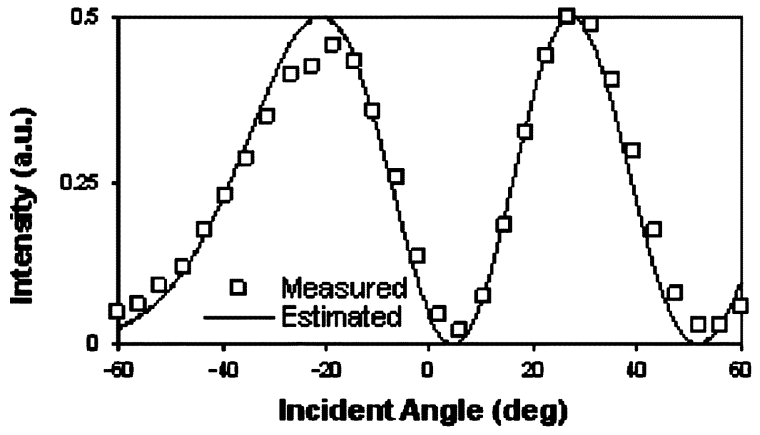

(c)

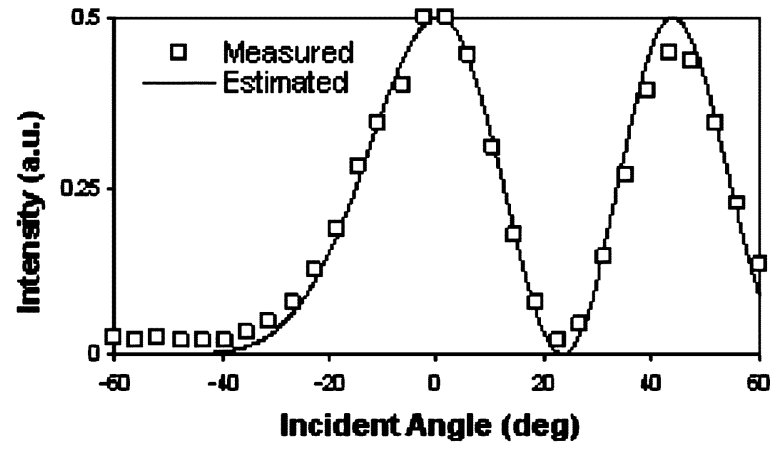

(b)

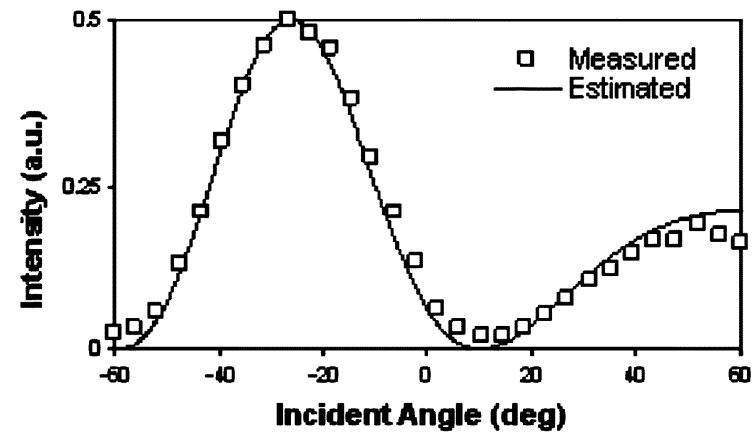

(d)

Fig. 6. Transmittance versus incident angle of samples: (a) A; (b) B; (c) C; (d) D.

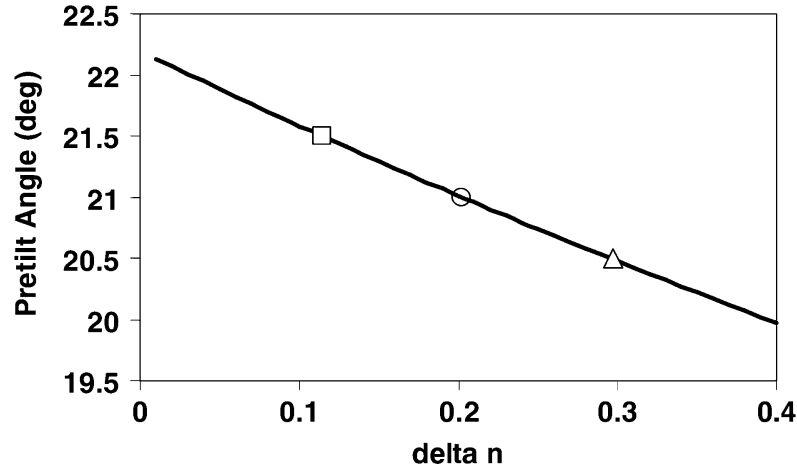

Fig. 7. Dependence of pretilt angle with birefringence $\Delta n$.

experiment data (square) are almost overlapped to the estimation (solid line). As the sampling area of the test cell is around $6 \mathrm{~mm}$ for $\pm 60^{\circ}$ scanning, the small deviation may come from the nonuniformity of the LC cell.

\section{DISCUSSION}

Furthermore, this method is insensitive to slight uncertainties in $n_{o}$ and $n_{e}$ as well. It is because the pretilt angle is obtained from the ratio of retardations $\chi$ rather than the retardation value directly. Such effect is depicted in Fig. 7. The simulation parameters are cell gap $d=5 \mu \mathrm{m}, n_{e}=1.71, n_{o}=1.5076, \Delta n_{0}=$ 0.2024 , and the pretilt angle is $\alpha_{0}=21^{\circ}$ (circle point), which has a minimum tolerance $\sim 1 \%$ according to previous discussions.

From Fig. 7, it can be seen that the proposed method is not sensitive to the magnitude of $n_{o}$ and $n_{e}$. It can be found that, even if there is $45 \%$ deviation (square and triangle point) away from the $\Delta n_{0}$ (circle point), the error is less than $\pm 0.5^{\circ}$.

\section{CONCLUSION}

In summary, we have proposed and verified a novel method for the measurement of large pretilt angles in an LCD cell with small cell gap. The error of the method is less than $\pm 1^{\circ}$. It can measure a full range of pretilt angles, from $0^{\circ}$ to $90^{\circ}$. The additional merit of this method is that the cell gap can be determined at the same time while $n_{e}$ and $n_{o}$ are insensitive to the measurement. This method is also reasonably fast and amenable to automation. In principle, it can be used in conjunction with a detector array and an array of light sources to measure the spatial variation of the pretilt angle as well as the cell gap in a single measurement. Both transmissive and reflective cells can be measured.

\section{REFERENCES}

[1] F. S. Y. Yeung, J. Y. Ho, Y. W. Li, F.-C. Xie, O. Tsui, P. Sheng, and H.-S. Kwok, "Variable liquid crystal pretilt angles by nano-structured surfaces," Appl. Phys. Lett., vol. 88, pp. 051910-051912, 2006.

[2] F. S. Y. Yeung, Y. W. Li, and H. S. Kwok, "Pi-cell liquid crystal displays at arbitrary pretilt angles," Appl. Phys. Lett., vol. 88, pp. 041108-041110, 2006

[3] F. S. Y. Yeung and H. S. Kwok, "Fast-response no-bias-bend liquid crystal displays using nano-structured surfaces," Appl. Phys. Lett., vol. 88, pp. 063305-063307, 2006.

[4] Y. Satoh, H. Mazaki, E. Yoda, T. Kaminade, T. Toyooka, and Y. Kobori, "Comparison of nematic hybrid and discotic hybrid films as viewing angle compensator for NW-TN-LCDs," in SID Int. Symp. Dig. Tech. Papers., 2000, vol. 31, pp. 348-351.

[5] H.-S. Kwok, F. S. Y. Yeung, and Y. W. Li, "Bistable LCDs based on a new alignment technology," in SID Int. Symp. Dig. Tech. Papers, 2006, pp. $1622-1625$. 
[6] F. S.-Y. Yeung, Y.-W. Li, and H.-S. Kwok, "Dual-frequency bistable bend-splay LCDs," in SID Int. Symp. Dig. Tech. Papers, 2005, pp. $1770-1773$.

[7] M. P. Cuminal and M. Brunet, "A technique for measurement of pretilt angles arising from alignment layers," Liq. Crys., vol. 22, pp. 185-192, 1997.

[8] J. S. Gwag, S. H. Lee, K. H. Park, W. S. Park, K. Y. Han, C. G. Jhun, T. H. Yoon, J. C. Kim, D. M. Song, and D. M. Shin, "Simple method for measuring the high pretilt angle of nematic liquid crystals," J. Appl. Phys., vol. 93, pp. 4936-4938, 2003.

[9] P. W. Rhodes and D. L. Shealy, "Refractive optical systems for irradiance redistribution of collimated radiation: Their design and analysis," Appl. Opt., vol. 19, pp. 3545-3553, 1980.

[10] P. Yeh, "Extended Jones matrix method," J. Opt. Soc. Amer., vol. 72, pp. 507-513, 1982.

[11] A. Lien, "Extended Jones matrix representation for the twisted nematic 1 iquid-crystal display at oblique incidence," Appl. Phys. Lett., vol. 57, pp. 2767-2769, 1990.

[12] F. H. Yu and H. S. Kwok, "Comparison of extended Jones matrices for twisted nematic liquid crystal displays at oblique angles of incidence," J. Opt. Soc. Amer. A, vol. 16, pp. 2772-2780, 1999.

[13] E. Hecht, Optics. New York: Addison-Wesley, 2002.

[14] T. C. Oakberg, "Measurement of wave-plate retardation using a photoelastic modulator," Proc. SPIE, vol. 3121, pp. 19-22, 1997.

[15] Y. L. Lo and P. F. Hsu, "Birefringence measurements by an electrooptic modulator using a new heterodyne scheme," Opt. Eng., vol. 41, pp. 2764-2767, 2002.

[16] S. Nakadate, "High precision retardation measurement using phase detection of Young's fringes," Appl. Opt., vol. 29, pp. 242-246, 1990

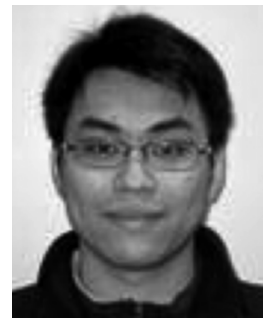

Y. W. Li received the B.Eng. and M.Phil. degrees in computer engineering from Hong Kong University of Science and Technology, Hong Kong, in 2001 and 2003 , respectively, where he is currently working toward the Ph.D. degree under the supervision of Prof. H. S. Kwok.

His current research interests include the study of fast-response liquid crystal displays (LCDs), bistable LCDs, and LCD characteristic measurements.

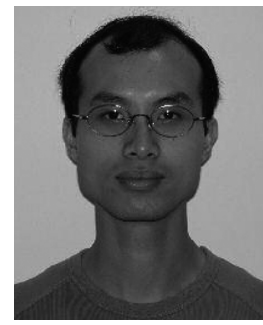

Jacob Y. L. Ho received the B.S. degree in engineering physics from the Hong Kong Polytechnic University, Hong Kong, in 1993, and the M.Phil. degree in physics and M.Sc. degree in electronic engineering from the Hong Kong University of Science and Technology (HKUST) in 1995 and 1999, respectively.

$\mathrm{He}$ is currently a Senior Technical Officer with the Department of Electronic and Computer Engineering, HKUST.

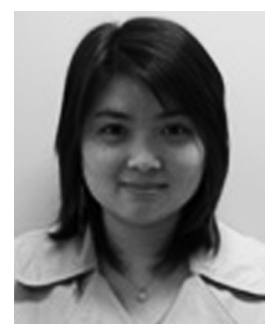

Fion S. Y. Yeung received the B.Eng. and M.Phil degrees from the Hong Kong University of Science and Technology, Hong Kong, in 2002 and 2004, respectively, where she is currently working toward the $\mathrm{Ph} . \mathrm{D}$. degree in electronic and computer engineering under the supervision of Prof. H. S. Kwok.

Her current research interest is in fast liquid crystal displays (LCDs) and bistable LCDs.

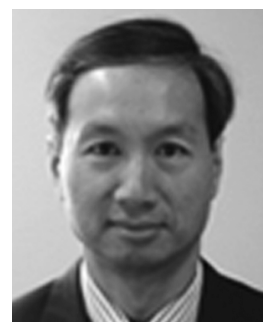

H. S. Kwok (S'73-M'78-SM'84-F'01) received the Ph.D. degree in applied physics from Harvard University, Cambridge, MA, in 1978.

$\mathrm{He}$ was a Professor of Electrical and Computer Engineering with the State University of New York, Buffalo, from 1980 to 1992 . He joined the Hong Kong University of Science and Technology, Hong Kong, in 1992. He has published over 500 papers and holds 25 patents in optics and liquid crystal display technologies.

Dr. Kwok is a Fellow of the Optical Society of America and the Society for Information Display. He was the recipient of the U.S. Presidential Young Investigator Award in 1984. 